\title{
Linhas Orientadoras para Pensar, Desenvolver e Implementar a Comunicação em Saúde em Portugal
}

\author{
Guidelines to Think, Develop and Implement Health \\ Communication in Portugal
}

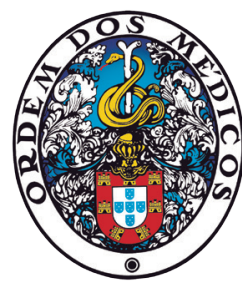

\author{
Débora MIRANDA ${ }^{1}$, Isa GALHORDAS ALVES ${ }^{2}$, Marta SALAVISA ${ }^{3}$ \\ Acta Med Port 2021 Oct;34(10):698-706 - https://doi.org/10.20344/amp.15770
}

\section{RESUMO}

A pandemia pelo vírus SARS-CoV-2 expôs e agravou muitas fragilidades dos sistemas de saúde, algumas já identificadas por organizações internacionais, nomeadamente as carências de meios materiais e humanos ou o insuficiente envolvimento da sociedade civil nos processos de decisão. À medida que as consequências socioeconómicas da pandemia se agravam e a fadiga pandémica se instala, outra fragilidade é posta a descoberto no espaço público e mediático: a falta de uma massa crítica de comunicação em saúde, com consequências diretas na gestão da pandemia. Comunicar neste contexto de crise e incerteza exige identificar os factos importantes a comunicar a diferentes segmentos da população, clarificar mitos e informações falsas, caracterizar as incertezas relevantes, ouvir e envolver os stakeholders e os cidadãos, desenvolver e testar as mensagens e monitorizar e avaliar as estratégias implementadas. A utilização da comunicação enquanto estratégia de apoio à gestão torna as organizações mais capazes de construir confiança, criar reputação, gerir relações com stakeholders internos e externos e de se prepararem para as incertezas do futuro, competências fundamentais para gerir uma crise sanitária. Dada a escassez de evidência científica sobre a comunicação em saúde realizada em Portugal, parte significativa da análise e diagnóstico é baseada no conhecimento empírico das autoras, adquirido através da experiência profissional, na área da comunicação, em diversas instituições de saúde. No final do artigo, as autoras propõem linhas orientadoras para pensar, desenvolver e implementar a comunicação em saúde em Portugal, nomeadamente numa crise sanitária, sustentadas numa cultura de liderança, colaboração e confiança.

Palavras-chave: Colaboração Intersetorial; Comunicação em Saúde; Liderança; Portugal

\section{ABSTRACT}

Sudden The SARS-CoV-2 pandemic has laid out and deepened several weaknesses of health systems, some of which have already been identified by international organizations, such as the lack of human and material resources or civil society's insufficient involvement in decision-making processes. As the pandemic's socioeconomic consequences worsen and pandemic fatigue settles in, another frailty is unveiled to the public and media space: the lack of a critical mass of health communicators, which has direct consequences on how the pandemic is managed. Communicating in such a context of crisis and uncertainty requires identifying what are the important facts to communicate to different population segments, clarifying myths and fake information, responding to ongoing uncertainties, listening to and involving stakeholders, developing and testing messages, and monitoring and evaluating implemented strategies. The use of communication as a management support strategy allows organizations to build confidence and reputation, manage relationships with internal and external stakeholders, and prepare for future uncertainties, which are crucial competencies in managing a health crisis. Given the lack of scientific evidence about health communication practiced in Portugal, a significant part of the analysis and diagnosis is based on the authors' empirical knowledge, obtained through professional experience in communications at several health institutions. At the end of the article, the authors propose guiding principles to think through, develop and implement health communication in Portugal, particularly during a health crisis, supported by a culture of leadership, collaboration, and confidence.

Keywords: Health Communication; Intersectoral Collaboration; Leadership; Portugal

\section{O DESAFIO}

\section{Comunicar em tempo de crise sanitária}

A pandemia pelo vírus SARS-CoV-2 veio exigir dos governos e das instituições de saúde o desenvolvimento de estratégias de comunicação complexas, que visam informar de forma clara e eficaz, minimizar o medo excessivo, gerir a infodemia ${ }^{1}$ e contribuir para aumentar a adesão dos cidadãos às medidas preventivas recomendadas.

O desafio em si já era extremamente exigente, mas a dificuldade foi potenciada por se tratar de um novo coronavírus, cujo comportamento e impacto na saúde a longo prazo permanece por esclarecer, o que aumentou o nível de incerteza e colocou obstáculos adicionais à comunicação precisa dos riscos associados e das medidas adequadas.
Comunicar em contexto de pandemia, nesta conjuntura, exige uma mudança urgente de estratégia e a adoção de uma cultura de liderança colaborativa, que implica envolver, de forma inclusiva, equipas alargadas de stakeholders, recorrendo à inteligência coletiva, para encontrar as melhores soluções. ${ }^{2}$ Mais do que nunca, é prioritário o trabalho em rede para se encontrarem respostas partilhadas, articulando diferentes áreas do conhecimento especializado, mas também aqueles que são diretamente impactados pela pandemia, trabalhando de forma próxima com as populações e comunidades.

O desenvolvimento das estratégias de comunicação mais adequadas exige a constituição de uma massa crítica

1. European Public Health Association. Países Baixos e Portugal.

2. Consultora Independente. Isa Alves - Consultoria de Comunicação, Lda. Portugal.

3. Gabinete de Comunicação e Marketing. Escola Nacional de Saúde Pública. Universidade NOVA de Lisboa. Lisboa. Portugal.

$\triangle$ Autor correspondente: Débora Miranda. debbiemiranda@gmail.com

Recebido: 20 de janeiro de 2021 - Aceite: 30 de abril de 2021 - First published: 24 de agosto de 2021 - Online issue published: 01 de outubro de 2021 Copyright @ Ordem dos Médicos 2021 
de comunicação, dotada de competências específicas em comunicação, mas também de outras, nomeadamente a capacidade de mobilizar e trabalhar em equipas alargadas, que facilitem a ativação de respostas céleres, informadas e inovadoras.

\section{O ECOSSISTEMA DA COMUNICAÇÃO EM SAÚDE EM PORTUGAL: ALGUMAS CONSIDERAÇÕES}

Liderança e comunicação nas organizações: duas faces da mesma moeda

Existe uma forte relação entre liderança e comunicação. ${ }^{3}$ A comunicação é considerada uma competência fundamental para uma liderança eficaz. As organizações mais eficazes utilizam a comunicação como estratégia de apoio à gestão.

Compete às equipas de comunicação das organizações de saúde o apoio de comunicação nas suas diferentes vertentes (institucional, em saúde, de crise e de risco, em emergências de saúde pública), sendo esta função transversal a todas as atividades, nas suas diversas fases (planeamento, desenvolvimento, implementação e avaliação).

Da experiência das autoras, a liderança nas organizações de saúde em Portugal segue por vezes um modelo muito hierarquizado e que não privilegia verdadeiramente a comunicação, encarando-a apenas como uma atividade técnica de suporte, sem reconhecer a sua função estratégica de apoio à gestão. É frequente os profissionais de comunicação não estarem envolvidos desde o início dos processos, sendo habitualmente chamados 'à mesa' apenas no momento em que é necessário comunicar para o exterior.

No contexto da pandemia de COVID-19, apenas dois dos 68 membros da task-force de resposta criada pela Direção-Geral da Saúde (DGS), são especialistas na área da comunicação. ${ }^{4}$

Este é um problema antigo e cultural na liderança das organizações. Apesar do aparente crescimento do reconhecimento da comunicação nas instituições de saúde em Portugal, evidenciado pelas recentes contratações de profissionais desta área ou pela criação, pela primeira vez em 2018, de uma Divisão de Comunicação e Relações Públicas na Direção-Geral da Saúde, ${ }^{5}$ continua a existir um desconhecimento acerca do seu potencial estratégico.

\section{Falta sistémica de investimento na comunicação em saúde}

Ao longo dos últimos 15 anos, as autoras observaram, no âmbito do seu percurso profissional, uma carência sistémica de investimento nas funções de comunicação em muitas organizações de saúde em Portugal. As equipas de comunicação encontram-se muitas vezes subdimensionadas, desprovidas de orçamento próprio e dedicado para as suas atividades, e nem sempre dispõem de tecnologias de comunicação sofisticadas.

As restrições na contratação de recursos humanos na administração pública constituem uma limitação ao recrutamento de especialistas em comunicação. Apesar de ser cada vez menos comum, existem ainda casos em que as funções da comunicação são exercidas por técnicos sem competências nesta área.

Adicionalmente, não existe, na administração pública, um investimento consistente na atualização dos conhecimentos em comunicação dos profissionais. A Direção-Geral da Qualificação dos Trabalhadores em Funções Públicas (INA) oferece um módulo transversal de conteúdos generalistas em 'Comunicação e Marketing', adequado a profissionais de outras áreas, mas que não serve o propósito de uma atualização adequada nesta área de especialidade em constante mutação.

\section{Equipas de comunicação em saúde}

Nos últimos anos houve um aumento de contratações de jornalistas para funções nas equipas de comunicação das instituições de saúde, no setor público. Estes profissionais aliam o conhecimento sobre o funcionamento dos órgãos de comunicação social à compreensão teórica e técnica sobre o setor da saúde, área onde se especializaram a fazer jornalismo e estão aptos a descodificar o vocabulário, conceitos e jargão técnico dos especialistas.

A assessoria de imprensa é um instrumento que medeia a relação entre as instituições e os órgãos de comunicação social, canais privilegiados para a comunicação massificada com as suas audiências. Contudo, as funções de comunicação das instituições de saúde têm de responder a desafios mais amplos.

É necessário garantir que as equipas de comunicação detêm um conjunto de competências adequadas para desempenhar eficazmente as suas funções. Com base no acima exposto, no final do artigo é sugerida uma proposta com as aptidões que consideramos necessárias para a constituição de uma massa crítica para a comunicação em saúde (Tabela 1).

\section{Estratégias de comunicação em saúde baseadas em evidência}

Autoridades governamentais e de saúde de todo o mundo têm utilizado estratégias de comunicação com o objetivo de informar, motivar e mobilizar a população no combate à pandemia. Portugal não foi exceção. As autoridades têm promovido, nas diferentes fases da pandemia, campanhas de comunicação com o objetivo de informar e influenciar comportamentos, que abrangem diversos temas, desde a promoção da lavagem das mãos e outros comportamentos preventivos, à mobilização da população para a adesão à vacinação.

Campanhas de mass media bem desenhadas, com mensagens consistentes e implementadas com intensidade suficiente, durante períodos de tempo sustentados, podem contribuir para o aumento da literacia em saúde, influenciar atitudes e crenças e contribuir para a mudança de comportamentos, ${ }^{6,7}$ nomeadamente quando integradas num conjunto mais vasto de intervenções (disponibilização de álcool-gel, apoios económicos para quem presta assistência a familiares com COVID-19, ou a aplicação de 
Tabela 1 - Proposta de perfil para a massa crítica de Comunicação em Saúde

\begin{tabular}{ll}
\hline Atividade profissional & Assessor/ Consultor/ Técnico/ Diretor de Comunicação \\
\hline Funções & Apoio de comunicação nas diferentes vertentes (institucional, em saúde, de crise e de risco \\
em emergências de saúde pública). Aconselhamento estratégico aos decisores. & Social listening, análise e antecipação de tendências. \\
& Produção de conteúdos. \\
& Visão de 360 graus das organizações com as quais colabora. \\
Elo de ligação entre diferentes departamentos e stakeholders (internos e externos). & Capacidade para mobilizar (não influenciar) a participação. Diplomacia. \\
Competências de comunicação & Transparência e honestidade a comunicar. \\
e liderança & Capacidade para gerir a incerteza e a complexidade. \\
& Trabalhar em redes alargadas e colaborativas de stakeholders. \\
& Autonomia para a tomada de decisão. \\
& Orientação para resultados. \\
& Storytelling. \\
Domínio de técnicas de monitorização e avaliação. & \\
Coragem, assertividade e resiliência. & Ética e honestidade. \\
Agilidade de aprendizagem. \\
Aptidões colaborativas. \\
Capacidade de resolução de problemas complexos de forma rápida e ágil.
\end{tabular}

sanções no caso de incumprimento em situações de alerta, contingência ou calamidade decretadas pelo governo).

Apesar de estarem disponíveis meta análises e estudos sobre a eficácia de intervenções de comunicação em saúde, nomeadamente campanhas de mass media, ${ }^{8}$ grande parte das campanhas de comunicação em saúde em Portugal não são baseadas em evidência.

A utilização de estratégias de apelo ao medo, através de imagens chocantes, por exemplo, ou à culpabilização, onde a mensagem é passada num tom acusatório, são regularmente adotadas por publicitários nas campanhas de saúde pública, em especial rodoviárias e antitabágicas, tendo sido igualmente utilizadas no âmbito da COVID-19. ${ }^{9}$

No entanto, os efeitos destas estratégias estão estudados e há evidência que demonstra que a sua utilização não é a mais indicada no contexto da pandemia. ${ }^{10}$ Estratégias de comunicação que promovem mecanismos cognitivos e comportamentais para lidar com situações adversas, e que estimulam a auto-eficácia - como a campanha da DGS "Seja um agente de Saúde Pública"- são consideradas mais eficazes para a alteração de comportamentos em saúde. ${ }^{11}$

Adicionalmente, a utilização de estratégias de indução de medo e de culpabilização, além de eticamente questionável, ${ }^{12}$ cria resistências na relação entre as instituições e os cidadãos, quando é especialmente importante reforçar a confiança, com vista à colaboração. ${ }^{13-15}$

Finalmente, a implementação de intervenções em saúde precisa de ser acompanhada de modelos de avaliação e monitorização. A ausência de uma cultura de avaliação adequada, com métricas e indicadores, não permite conhecer a eficácia das ações e campanhas de comunicação. Tal representa um problema adicional para a tomada de decisão baseada em dados (data-driven).

\section{BOAS PRÁTICAS DE COMUNICAÇÃO EM SAÚDE, SO- BRETUDO EM TEMPOS DE PANDEMIA Trabalho em rede}

A comunicação em saúde não se faz apenas com profissionais de comunicação. A definição de estratégias de comunicação em saúde eficazes compreende não só o domínio de áreas do conhecimento como a assessoria de imprensa, comunicação de crise, de risco, comunicação clínica, de ciência, marketing social, literacia em saúde, ${ }^{16}$ mas pressupõe também a articulação com outras disciplinas e setores e o trabalho em rede com diferentes stakeholders, o que exige a negociação de diferentes pontos de vista. ${ }^{17}$

Os stakeholders contribuem com capital intelectual, recursos materiais, humanos e financeiros e ligações a redes de contactos mais alargadas. Trabalhar em redes colaborativas permite incluir maior diversidade de ideias e perspetivas nos processos de tomada de decisão e contribui para uma operacionalização mais eficiente das estratégias de comunicação, já que os stakeholders serão aliados na execução das ações que eles próprios ajudaram a definir.

Às autoridades responsáveis pela gestão da pandemia compete a liderança deste processo, mas a qualidade dessa liderança é determinada pela capacidade de trabalhar em rede e comunicar, de forma eficaz, neste "ecossistema" de diferentes atores, no âmbito do qual é também fundamental envolver as populações e as comunidades. ${ }^{18}$

Assim sendo, a comunicação, nestes processos, tem de reunir alguns requisitos fundamentais: partir de um exercício de escuta, ser honesta, clara, igualitária, diplomática e não-autoritária ou não-coerciva. Follet definiu o conceito da partilha de poder não-coerciva, defendendo que os líderes têm "poder com", isto é, um poder que é desenvolvido em conjunção, e não "poder sobre" as equipas com quem trabalham. ${ }^{19}$ Neste tipo de liderança, os diferentes atores críticos recrutados para uma determinada missão sentam-se à mesa e debatem ideias, co-construindo soluções em 
função de objetivos e resultados partilhados.

\section{A confiança no centro da comunicação com o cidadão}

Planear a comunicação, de forma estratégica, durante uma pandemia envolve auscultar todos os envolvidos, definir objetivos, implementar de forma coordenada e participada as intervenções direcionadas para diferentes segmentos e monitorizar e avaliar as atividades, procurando melhorar a sensibilização pública e influenciar os comportamentos, antes, durante e depois da pandemia. Idealmente, este planeamento deverá começar a ser estruturado, de forma proativa, antes ainda da ocorrência de uma emergência de saúde pública.

Contudo, a confiança dos cidadãos nas organizações de saúde é o primeiro requisito para assegurar o sucesso da comunicação e, consequentemente, o cumprimento das medidas de prevenção. Sem confiança, o planeamento será insuficiente. Segundo a Organização Mundial da Saúde (OMS), há três pilares fundamentais para construir esta relação de confiança com a população numa emergência de saúde pública: comunicar o risco eficazmente, reconhecer a incerteza e envolver as pessoas e as comunidades. ${ }^{20}$

\section{Comunicar o risco e a incerteza}

É necessário que as populações conheçam os riscos e os comportamentos que devem adotar para se protegerem e às suas famílias, em contexto de pandemia. A comunicação de risco consiste na troca de informação, recomendações e opiniões em tempo real entre especialistas, líderes comunitários ou autoridades e as pessoas em risco. Deve ser precedida por uma auscultação das preocupações e necessidades dos cidadãos e procurar manter sempre canais de diálogo abertos.

As autoridades de saúde devem reconhecer, de forma transparente e honesta, a existência de incerteza associada aos riscos (incluindo quando é necessário reportar informação negativa, como o número de mortes) e assumir o que se sabe e não se sabe nas diferentes fases da pandemia. Esta comunicação dos riscos e da incerteza deve ocorrer o mais precocemente possível e ser realizada de forma explícita, não contraditória e clara, sem recorrer a termos técnicos. ${ }^{20}$

A literatura mostra que o recurso a narrativas (storytelling) para explicar a incerteza científica melhora a compreensão, o interesse e o envolvimento dos públicos não especializados. ${ }^{21,22}$ Muitos profissionais de saúde e cientistas resistem ao uso destas técnicas para comunicar resultados em ciência, pois acreditam que estão associadas a perda de rigor, sendo difícil traduzir a complexidade inerente à ciência experimental para formatos narrativos. ${ }^{23}$ Contudo, as narrativas são fundamentais para estabelecer ligações entre conceitos científicos, muitas vezes incompreensíveis para as audiências, e as suas experiências de vida quotidiana. ${ }^{21} \mathrm{O}$ recurso a metáforas, analogias ou comparações com riscos que sejam percetíveis para a população pode ser particularmente útil para comunicar situações de risco e incerteza, melhorando a compreensão das mensagens e proporcionando uma ligação emocional. A emoção funciona como um atalho que facilita o processamento e a retenção da informação, bem como a melhor compreensão dos riscos envolvidos, motivando as pessoas para a ação de forma mais efetiva.

O recurso a técnicas narrativas é ainda útil para a apresentação de informação numérica e estatística complexa, em particular junto de audiências com baixa literacia numérica e em saúde, que são significativas em Portugal. ${ }^{21,22,24}$

Adicionalmente, na comunicação de risco é essencial assegurar a coordenação das mensagens a disseminar. Para isso, as parcerias com os stakeholders, incluindo os media, são fundamentais, possibilitando veicular mensagens uniformes em canais diversos. Só assim será possível minimizar a disseminação de informação conflituante entre diferentes porta-vozes.

\section{Envolver as populações e as comunidades}

Promover a participação das populações e as comunidades nos processos de comunicação é essencial para assegurar a sua colaboração e a adequação das medidas aos seus diferentes contextos de vida. Para isso, é necessário identificar os parceiros e líderes comunitários em quem estas confiam, mobilizando-os e envolvendo-os nas tomadas de decisão. ${ }^{18}$

O envolvimento dos parceiros locais na definição das medidas e mensagens a comunicar assegura o fluxo de informação entre diversos setores e a diferentes níveis, bem como a consistência da informação e a articulação entre os porta-vozes, desde os primeiros momentos da pandemia. Adicionalmente, promove uma cultura de co-responsabilização entre todos os intervenientes e, caso seja necessário adotar mecanismos de correção ou adicionar elementos novos à comunicação, a resposta a essas necessidades tenderá a ser bastante mais rápida e conforme.

As mensagens têm de ser co-construídas com as comunidades, adaptadas aos seus contextos culturais e às suas necessidades de informação, e pré-testadas com audiências selecionadas. Devem igualmente ser revistas e alteradas sempre que necessário no decurso da evolução da pandemia. A informação deve ser veiculada com recurso aos canais de comunicação mais adequados, indo ter com as pessoas onde estas se encontram e consomem informação.

\section{O exemplo da infodemia em tempo de COVID-19}

A Organização Mundial de Saúde afirmou, em fevereiro de 2020, que não estamos a combater apenas uma pandemia mas também uma infodemia. ${ }^{25} \mathrm{~A}$ infodemia é a superabundância de informação, online e offline, que pode incluir desinformação, má informação, informação falsa, rumores e teorias da conspiração, entre outros. Uma infodemia inclui tentativas deliberadas de disseminar informação incorreta para enfraquecer a resposta de saúde pública e promover agendas alternativas de determinados grupos ou indivíduos. Em última análise, pode prejudicar a saúde física e mental e aumentar o estigma sobre determinados 
grupos. ${ }^{26}$

A infodemia não é um fenómeno novo mas, na era digital em que vivemos, dissemina-se em tempo real e abre terreno para incertezas, impedindo os cidadãos de digerir e analisar de forma crítica a informação que consomem. Além disso, episódios associados à COVID-19, como por exemplo declarações oficiais utilizadas fora de contexto, podem comprometer a confiança nas autoridades de saúde. ${ }^{27}$ Ora, sendo a confiança o primeiro requisito para assegurar o sucesso da comunicação, a falta dela pode refletir-se numa não adesão das pessoas às medidas de prevenção. Gerir a infodemia atual requer, portanto, uma recuperação da confiança nas autoridades de saúde (como ministérios da saúde) e nos seus líderes, mensagens e canais de informação. ${ }^{28}$

Por exemplo, as populações jovens têm, historicamente, baixos níveis de confiança nas autoridades. ${ }^{29} \mathrm{E}$, apesar do seu menor risco de letalidade por COVID-19, têm um elevado risco de propagar o vírus. A sua motivação para adotar um comportamento preventivo dependerá da confiança que depositam no canal ou no mensageiro que lhes faça esse pedido.

O desafio de comunicar durante uma crise sanitária e, simultaneamente, de infodemia passa por conjugar a verdade com a oportunidade. Por um lado, comunicar a evidência científica correta e promover a adoção de comportamentos preventivos. Por outro, reconhecer que aqueles que propagam rumores e informações falsas não sentem a necessidade de aceder a dados e rigor científico antes de o fazer. Para preencher o vazio de respostas que as pessoas legitimamente procuram, as autoridades de saúde pública têm de comunicar mais depressa. ${ }^{30}$ Caso contrário, o vazio será preenchido por desinformação.

Como vimos, o uso de estilos narrativos (storytelling) e outras técnicas persuasivas podem ajudar a explicar o risco e a incerteza científica, que são conceitos complexos, pouco imaginativos e do âmbito cognitivo. No caso da infodemia, os rumores, o estigma e as teorias da conspiração são também narrativas, de âmbito emocional, que muitas vezes se sobrepõem aos factos científicos. Além disso, referir uma informação falsa, mesmo que seja para corrigi-la, pode ter o efeito perverso de reforçá-la.

Também na infodemia, a chave para a sua gestão - e aquilo que tem escasseado e levado a desinformação a bom porto - é a cultura colaborativa. Não é suficiente comunicar uma mensagem; é necessário desenvolver intervenções multidisciplinares e multisetoriais que envolvam política, sociedade civil, comunidades locais, indivíduos, sistemas de saúde, comunicação social, empresas de tecnologia, ciências sociais, entre outros, que permitam equilibrar a esperança e a preocupação. ${ }^{31}$ Por sua vez, este problema global sem precedentes exige soluções locais que possam ser alavancadas por líderes comunitários como professores, padres, figuras públicas, cientistas, etc.

$O$ reconhecimento de que a infodemia em contexto digital é um fenómeno novo levou a OMS a liderar uma formação à escala global ${ }^{32}$ que pretende justamente fomentar a colaboração multidisciplinar nesta área, permitindo que agentes de diversos setores e culturas tenham uma rede internacional onde partilhar as suas experiências, conhecimento e soluções locais.

Assume-se que é o acesso à informação que leva as pessoas a adotar comportamentos saudáveis. Mas gerir uma infodemia exige compreender, mais do que como a informação flui, os pensamentos e comportamentos das pessoas. Também neste contexto, o cidadão deve estar no centro de todas as intervenções de gestão de uma infodemia. Embora as redes sociais deem, muitas vezes, eco às vozes mais ruidosas, estas não representam necessariamente a maioria. Para compreendermos a diversidade de perceções de risco e encontrarmos soluções direcionadas, é necessário auscultar a sociedade (social listening). ${ }^{30}$

Uma vez compreendidas as perceções sociais, devemos então levar a cabo a boa prática de envolvimento das populações no desenvolvimento das mensagens, chamando à mesa da discussão os seus destinatários. Também

Tabela 2 - Proposta de perfil do Gestor de Infodemia

\begin{tabular}{|c|c|}
\hline Atividade profissional & Gestor (ou equipa de gestão) de Infodemia \\
\hline Funções & $\begin{array}{l}\text { Promoção (ou recuperação) de confiança nas autoridades de saúde. } \\
\text { Envolvimento dos stakeholders dos diversos setores relevantes, sobretudo da sociedade civil. } \\
\text { Inoculação, isto é, tornar as populações resilientes à informação falsa. } \\
\text { Monitorização de informação e de impacto das intervenções. } \\
\text { Desconstrução de desinformação em caso de perigo para a saúde. }\end{array}$ \\
\hline Características & $\begin{array}{l}\text { Atitude democratica. } \\
\text { Capacidade para digerir evidência de múltiplas disciplinas. } \\
\text { Capacidade para identificar oportunidades e agir proativamente num curtoespaço de tempo. } \\
\text { Equilíbrio entre a ciência e a ação. }\end{array}$ \\
\hline $\begin{array}{l}\text { Competências de comunicação } \\
\text { e liderança }\end{array}$ & $\begin{array}{l}\text { Coerência. } \\
\text { Espírito colaborativo. } \\
\text { Gestão de projeto. } \\
\text { Compromisso pela ciência e pelo bem comum. }\end{array}$ \\
\hline Aptidões pessoais & $\begin{array}{l}\text { Calma e proatividade. } \\
\text { Influência, acessibilidade e confiança. } \\
\text { Respeito por diferentes culturas. } \\
\text { Sentido cívico. }\end{array}$ \\
\hline
\end{tabular}


aqui encontramos boas práticas de liderança, que passam por uma escuta empática das preocupações e dúvidas das pessoas e não por uma abordagem normativa. Neste contexto, é proposta no final do artigo a súmula das características específicas da pessoa ou equipa que gere uma infodemia (Tabela 2).

\section{RECOMENDAÇÕES PRÁTICAS}

Face ao exposto, propomo-nos apresentar linhas orientadoras para pensar, desenvolver e implementar a comunicação em saúde em Portugal, nomeadamente numa crise sanitária, sustentadas em princípios de liderança, cultura colaborativa e confiança.

As recomendações que se seguem decorrem das boas práticas e evidência existente em comunicação, bem como da experiência profissional das autoras.

\section{- Quais são as aptidões necessárias à constituição} de uma massa crítica de comunicação em saúde?

Estas poderão ser características reunidas numa só pessoa - tipicamente na função de diretor de comunicação - ou de uma equipa interna ou alargada, no caso em que se potenciam esforços sinérgicos em rede com parceiros.

\section{- Como constituir e gerir uma rede colaborativa de stakeholders?}

Implica adotar uma filosofia colaborativa e instituir processos sistemáticos para a resolução ágil de problemas, definindo objetivos, metas e responsabilidades. Neste modelo, é necessário desagregar problemas complexos em etapas críticas (por exemplo: cuidados intensivos, vacinação, doentes crónicos, campanha de comunicação, etc.), para dar resposta aos vários desafios.

- Envolver stakeholders: Identificar, o mais precocemente possível, os atores relevantes de vários setores e disciplinas para, em conjunto com as autoridades de saúde, co-criarem as soluções necessárias.

- Acordar um 'contrato social': As normas e comportamentos esperados que devem nortear o processo colaborativo (como a comunicação transparente, a integridade, a confidencialidade, a confiança, diferentes níveis de responsabilidade, etc.).

- Reunir stakeholders por etapa: Cada reunião terá uma agenda concreta, com questões específicas para as quais se procuram soluções. A documentação deve ser remetida com antecedência para permitir refletir e envolver antecipadamente as redes colaborativas.

- Dinamizar grupos de discussão: As reuniões devem iniciar-se em grande grupo, harmonizando em conjunto os objetivos de trabalho e responsabilidades. Divide-se depois em sub-grupos de cinco participantes, de modo a assegurar a efetiva participação. De regresso ao grande grupo, cada sub-grupo apresenta os seus resultados para a obtenção de feedback.

- Delinear projeto: Finalizadas as reuniões, as pro- postas tomam a forma de projeto, com tarefas e responsabilidades bem definidas. Ao fim de cada período de análise, a pessoa responsável por cada etapa deve reportar às respetivas equipas o que foi alcançado, dificuldades sentidas, apoios a solicitar e, se necessário, redefinir os compromissos para a fase seguinte.

- Como fomentar a confiança na comunidade científica, nos profissionais de saúde e nas instituições de saúde?

A adoção de comportamentos dependerá da credibilidade percecionada das autoridades de saúde que os defendem. Para isso, é necessário promover (ou recuperar) a confiança dessas autoridades, por sua vez alicerçada por uma estratégia de comunicação que cumpra os seguintes requisitos:

- Falar com honestidade: Os cidadãos não cumprem normas ditadas por pessoas/instituições em quem não confiam e, por sua vez, não confiam em pessoas que percecionam como sendo desonestas.

- Comunicar a incerteza: Quando um facto não é conhecido, deve assumir-se que não se sabe e porquê; a literacia científica passa por explicar às pessoas que a ciência é um processo e não uma verdade absoluta.

- Comunicar atempadamente: Não deixar perguntas por responder, sob pena de essas lacunas serem preenchidas por informação falsa.

- Melhorar as competências de comunicação: Investir nos currículos de comunicação (clínica, media training e não só). Investir em equipas de comunicação, e suas adequadas competências, nas instituições.

- Envolver as comunidades: Ouvir as preocupações e medos das pessoas com empatia, afinal, são os seus comportamentos que queremos influenciar. No caso do vírus do Ébola, as equipas de controlo da epidemia adaptaram os seus procedimentos de segurança e higiene de acordo com a cultura e costumes locais de prática do luto. ${ }^{33}$ As comunidades que se sentem ouvidas confiarão mais facilmente em quem as ouve.

- Como criar intervenções de comunicação em saúde pública eficazes e adequadas à população?

- Escutar com empatia: Se conseguirmos colocar-nos no papel das pessoas com quem queremos comunicar, desde o início do processo, conseguiremos dar respostas mais adequadas às suas necessidades.

- Colocar as questões certas: Se direcionarmos a pergunta para respostas que pensamos saber de antemão, arriscamo-nos a perder a oportunidade de chegar às verdadeiras motivações das pessoas. Por exemplo, antes de perguntar às pessoas se querem tomar a vacina contra a COVID-19, devemos 
perguntar o que as preocupa e se já tiveram COVID-19.

- Explicar os factos: Investir tempo em esclarecer as comunidades pode resolver mal-entendidos. Por exemplo, tal como foi imprescindível treinar as equipas de vacinação contra o Ébola a comunicar risco, respeitar as crenças locais e responder às dúvidas das populações, no caso da vacina para a COVID-19 devemos também ouvir as preocupações das pessoas, explicar em que estado de desenvolvimento ou de distribuição é que se encontra, quem lidera o processo, o seu grau de seguranca, etc.

- Incluir técnicas narrativas: Formatos de comunicação informativos e estatísticos são insuficientes para transmitir informação à população e, em particular, influenciar comportamentos. Por exemplo, o recurso a técnicas narrativas permite explicar a incerteza científica e melhorar a compreensão e o interesse dos públicos não especializados.

- Testar mensagens e materiais de comunicação: No desenvolvimento de mensagens e formatos destinados aos cidadãos, é imprescindível recrutar um segmento dessa população, ainda que pequeno, ouvir a sua opinião e implementar melhorias em conformidade.

\section{- Como contribuir para o combate à infodemia}

Em vez de ambicionarmos travar por completo uma infodemia, devemos procurar "achatar a curva infodémica", desacelerando a propagação de informação incorreta e perigosa. O objetivo não é produzir mais informação, mas abordar os fatores ambientais e sociais que facilitam a propagação de informação falsa. Algumas técnicas a adotar são:

- Agregar stakeholders: Não é possível gerir uma infodemia sem envolver todos os atores e setores intervenientes, desde as entidades governamentais às comunidades locais, passando por empresas de tecnologia, cientistas de dados, epidemiologistas, jornalistas, etc.

- Desmistificar informação falsa: Apenas quando ela compromete a saúde pública (por exemplo, quando o então Presidente dos Estados Unidos sugeriu a injeção intravenosa de desinfetante), se a fonte de informação é potencialmente credível e se se difunde muito depressa. Caso contrário, muitas vezes a referência a mensagens falsas pode reforçá-las, chegando a pessoas que, de outra forma, nunca as teriam confrontado.

- Adotar a 'truth sandwich': Ao desmistificar uma informação falsa, começar e terminar com os factos científicos, de modo a reforçá-los perante a mentira.

- Inocular: Ao difundir a evidência científica e explicar os mecanismos por detrás da infodemia, conseguimos tornar as comunidades mais resilientes perante futuras ameaças informativas.

- Atuar onde as pessoas estão: Os esforços de de- saceleração da informação falsa devem ser feitos nos mesmos canais onde ela é propagada e não necessariamente em canais "oficiais" que não têm a audiência mais relevante.

- Preencher o vazio: Esclarecer as dúvidas que as pessoas têm antes que sejam respondidas por fontes não credíveis. Ao mesmo tempo, devemos sensibilizar os meios de comunicação social a que, muitas vezes, se antecipam à evidência em prol do interesse público.

No seguimento da proposta de perfil para a massa crítica de comunicação em saúde (Tabela 1), apresentamos um conjunto de aptidões adicionais específicas dos profissionais responsáveis pela gestão de infodemia.

\section{- Como avaliar o impacto da comunicação?}

- Planear: Assegurar que cada intervenção tem um enquadramento de avaliação das ações de comunicação, para garantir a recolha de informação que permita monitorizar e analisar outcomes (resultados) e outputs (atividades desenvolvidas para atingir os resultados).

- Ver o que já foi feito (benchmark): Para definir o sucesso das ações de comunicação, há que ter pontos de comparação. A não ser que se esteja a implementar algo totalmente inovador e não haja comparação possível, é sempre viável consultar resultados de ações semelhantes no passado. Saber o que outros fizeram e como fizeram pode também ser muito útil para saber o que melhor resulta e/ou evitar cometer os mesmos erros.

- Ter dados baseline: Para saber se a comunicação teve impacto, é necessário saber como era o contexto antes da intervenção. É impossível avaliar sem ter dados baseline.

- Definir objetivos de comunicação: "De que forma pode a comunicação contribuir para os objetivos estratégicos?" é a pergunta a fazer para definir os objetivos de comunicação. É importante definir objetivos SMART (specific, measurable, achievable, relevant, timebound) e, para cada objetivo de comunicação, encontrar indicadores apropriados.

- Saber o que medir: Os indicadores de comunicação são estabelecidos na fase de planeamento das intervenções e devem refletir os diferentes níveis da ação de comunicação (atividades planeadas, outputs e outcomes expectáveis), o público-alvo, a sua dimensão e os canais de comunicação.

- Ser pragmático: Deverá procurar-se um equilíbrio entre o que é ideal medir e ajustar e o que é exequível. Por outro lado, deve evitar-se avaliar somente o que é fácil e conveniente.

\section{CONCLUSÃO}

A pandemia de COVID-19 é, em muitos aspetos, um fenómeno sem precedentes em todo o mundo. Assistimos a uma aprendizagem contínua em contexto de incerteza para 
governos, comunidade científica e cidadãos. No entanto, existe já muita evidência sobre o que é e não é eficaz e o que é necessário para implementar soluções com impacto. Lançamos assim uma last call to collaboration, uma última chamada para que colaboremos verdadeiramente, com confiança e sob uma liderança eficaz. Pretendemos acima de tudo apontar passos concretos para implementar soluções, capacitar instituições e profissionais, colocar a evidência em prática e aprender com os sucessos e os erros para estarmos melhor equipados para futuros desafios.

\section{CONTRIBUTO DOS AUTORES}

DM: Contribuição intelectual direta no desenho, redação e revisão do artigo, com especial enfoque no desenvolvimento do exemplo da temática da infodemia em tempos de COVID-19. Apreciação final e seleção das principais mensagens para a conclusão.

IG: Contribuição intelectual direta no desenho, redação e revisão do artigo, com especial enfoque nas temáticas relacionadas com a confiança no centro da comunicação, trabalho em rede e atributos para a constituição de uma massa crítica de comunicação em saúde.

MS: Contribuição intelectual direta no desenho, redação e revisão do artigo, com especial enfoque no desenvolvimento das temáticas relativas à comunicação e liderança nas organizações e estratégias de comunicação em saúde baseadas em evidência.

\section{CONFLITOS DE INTERESSE}

Os autores declaram a inexistência de conflitos de interesse na realização do presente trabalho.

\section{FONTES DE FINANCIAMENTO}

Este trabalho não recebeu qualquer tipo de suporte financeiro de nenhuma entidade no domínio público ou privado.

\section{REFERÊNCIAS}

1. World Health Organization. Call for action: managing the infodemic. [consultado 2021 jan 14]. Disponível em: https://www.who.int/news/ item/11-12-2020-call-for-action-managing-the-infodemic.

2. Surowiecki, J. The wisdom of crowds: why the many are smarter than the few and how collective wisdom shapes business, economies, societies, and nations. New York: Doubleday Books; 2004.

3. Zerfass A, Huck S. Innovation, communication, and leadership: new developments in strategic communication. Int $\mathrm{J}$ Strateg Commun. 2007;1:107-22.

4. Rajan D, Koch K, Rohrer K, Bajnoczki C, Socha A, Voss M, et al. Governance of the COVID-19 response: a call for more inclusive and transparent decision-making. BMJ Glob Health. 2020;5:e002655.

5. Portugal. Despacho n. ${ }^{\circ} 10312 / 2018$. Diário da República II Série, n. ${ }^{\circ}$ $214(2018 / 11 / 07)$. p.29868.

6. Wakefield M, Loken B, Hornik R. Use of mass media campaigns to change health behaviour. Lancet. 2010;376:1261-7.

7. Noar SM. A 10-year retrospective of research in health mass media campaigns: where do we go from here? J Health Commun. 2006;11:2142.

8. Anker A, Feeley T, McCracken B, Lagoe C. Measuring the effectiveness of mass-mediated health campaigns through meta-analysis. $\mathrm{J}$ Health Commun. 2016;21:439-56

9. Ruiter R, Kessels L, Peters G, Kok G. Sixty years of fear appeal research: current state of the evidence. Int J Psychol. 2014;49:63-70.

10. Stolow J, Moses L, Lederer A, Carter R. How fear appeal approaches in COVID-19 health communication may be harming the global community. Health Educ Behav. 2020;47:531-5.

11. van Bavel J, Baicker K, Boggio P, Capraro V, Cichocka A, Cikara M, et al. Using social and behavioural science to support COVID-19 pandemic response. Nat Hum Behav. 2020;4:460-71.

12. Hastings G, Stead M, Webb J. Fear appeals in social marketing: strategic and ethical reasons for concern. Psychol Mark. 2004;21:96186.

13. The University of British Columbia. Democratic health communications during Covid-19: a rapid response. [consultado 2021 mar 2]. Disponível em: https://democracy2017.sites.olt.ubc.ca/files/2020/09/DemocraticHealth-Communication-during-Covid_FINAL.pdf.

14. Toppenberg-Pejcic D, Noyes J, Allen T, Alexander N, Vanderford M, Gamhewage G. Emergency risk communication: lessons learned from a rapid review of recent gray literature on ebola, zika, and yellow fever. Health Commun. 2019;34:437-55.

15. Ontario Hospital Association. Effective communication strategies for COVID-19. [consultado 2021 mar 2]. Disponível em: https://www.oha. com/Documents/Effective $\% 20$ Communications $\% 20$ Strategies $\% 20$ for\%20COVID-19.pdf.

16. European Centre for Disease Prevention and Control. What is health communication? [consultado 2021 jan 14]. Disponível em: https://www. ecdc.europa.eu/en/health-communication/facts.

17. Mol A, Hardon A. What COVID-19 may teach us about interdisciplinarity. BMJ Glob Health. 2020;5:e004375.

18. Teixeira P, Vital Brito $D$, Araújo $R$, Gomes B. Risk communication and community engagement in the COVID-19 pandemic in Portugal. Acta Med Port. 2021;34:1-2.

19. Graham P. Mary Parker Follett - prophet of management: a celebration of writings from the 1920s. Boston: Harvard Business School Press; 1995.

20. World Health Organization. Communicating risk in public health emergencies: a WHO guideline for emergency risk communication (ERC) policy and practice. Geneva: WHO; 2017.

21. Dahlstrom $M$. Using narratives and storytelling to communicate science with nonexpert audiences. Proc Natl Acad Sci U S A. 2014;111:1361420.

22. National Academies of Sciences, Engineering, and Medicine. Communicating science effectively: a research agenda. Washington: The National Academies Press; 2017.

23. Katz Y. Against storytelling of scientific results. Nat Methods. 2013;10:1045

24. Espanha R, Ávila P, Mendes R. Literacia em Saúde em Portugal. Lisboa: Fundação Calouste Gulbenkian; 2016.

25. World Health Organization. Managing the COVID-19 infodemic: promoting healthy behaviours and mitigating the harm from misinformation and disinformation. [consultado 2021 jan 14]. Disponível em https://www.who.int/news/item/23-09-2020-managing-the-covid-19infodemic-promoting-healthy-behaviours-and-mitigating-the-harm-frommisinformation-and-disinformation.

26. The Lancet Infectious Diseases. The COVID-19 infodemic. Lancet Infect Dis. 2020;20:875.

27. Mheidly N, Fares J. Leveraging media and health communication strategies to overcome the COVID-19 infodemic. J Public Health Policy. 2020;21:410-20.

28. Tangcharoensathien V, Calleja N, Nguyen T, Purnat T, D'Agostino M, Garcia-Saiso S, et al. Framework for managing the COVID-19 infodemic: methods and results of an online, crowdsourced WHO technical consultation. J Med Internet Res. 2020;22:e19659.

29. Organisation for Economic Co-operation and Development. OECD policy responses to coronavirus (COVID-19) - youth and COVID-19: response, recovery and resilience; [consultado 2021 jan 14]. Disponível em: https://www.oecd.org/coronavirus/policy-responses/youth-andcovid-19-response-recovery-and-resilience-c40e61c6/\#biblio-d1e1597.

30. Eysenbach G. How to fight an infodemic: the four pillars of infodemic management. J Med Internet Res. 2020;22:e21820.

31. Gesualdo F, Bucci L, Rizzo C, Rozzi A. Digital tools, multidisciplinarity and innovation for communicating vaccine safety in the COVID-19 era. Hum Vaccin Immunother. 2021:1-4.

32. World Health Organization. $1^{\text {st }}$ WHO infodemic manager training [consultado $2021 \mathrm{abr}$ 26]. Disponível em: https://www.who.int/teams/ 
risk-communication/infodemic-management/1st-who-training-ininfodemic-management.

33. Lee-Kwan S, DeLuca N, Bunnell R, Clayton H, Turay A, Mansaray Y.
Facilitators and barriers to community acceptance of safe, dignified medical burials in the context of an ebola epidemic, Sierra Leone, 2014.

J Health Commun. 2017;22:24-30. 